Emília de Faria CARniel ${ }^{1}$

MARIA DE LURDES ZANOLLI

ANDRÉ MORENO MORCLLIO 3

Artigos originais

Palavras-chave

Cesárea/estatística \& dados numéricos Saúde sexual e reprodutiva Fatores socioeconômicos

Fatores de risco

Epidemiologia

Keywords

Cesarean section/statistics \& numerical data Sexual and reproductive health Socioeconomic factors

Risk factors

Epidemiology

\title{
Fatores de risco para indicação do parto cesáreo em Campinas (SP)
}

\author{
Risk factors for the indication of caesarean section \\ in Campinas (SP)
}

Resumo

OBJETIVO: conhecer a freqüência de cesarianas em Campinas (SP), e identificar fatores de risco para sua ocorrência. MÉTODOS: estudo transversal no qual se analisaram dados das Declarações de Nascidos Vivos de 2001. A variável dependente foi o tipo de parto e as variáveis independentes foram as características maternas, gestacionais, do parto e do recém-nascido. Na avaliação da associação entre variáveis empregou-se o teste do $\chi^{2}$ e calcularam-se valores de odds ratio (OR) brutos e ajustados. RESULTADOS: a taxa de cesáreas foi $54,9 \%$. As chances de cesárea foram aumentadas 1,9 vezes para mulheres de 20 a 34 anos (OR ajustado (ORaj)=1,9; IC a 95\%:1,7-2,1); 3,7 para as maiores de 35 anos (ORaj=3,8; IC a 95\%:3,2-4,5); 1,5 para as que estudaram até o ensino médio (ORaj=1,5; IC a 95\%: 1,4-1,6); 2,5 para as com ensino superior (ORaj=2,6; IC a 95\%:2,2-2,9); 1,3 para as com companheiro (ORaj=1,3; IC a $95 \%: 1,2-1,4) ; 1,6$ para as que trabalhavam $(O R a j=1,6$; IC a $95 \%: 1,5-1,8) ; 1,2$ para as que moravam em regiões com melhores Índices de Condição de Vida (ORaj=1,2; IC a 95\%: 1, O-1,3); 2,2 para as primíparas (ORaj=2,2; IC a $95 \%: 1,9-2,5) ; 1,6$ para as multíparas (ORaj=1,6; IC a 95\%: 1,4-1,9) e 3,1 vezes nas gestações duplas (ORaj=3,1; IC a 95\%:2,2-4,4). As mulheres com menos de sete consultas foram protegidas da cesárea $\mid O R a j=0,6 ; I C$ a 95\%:0,5$0,7)$. CONCLUSÕES: as chances para indicação de cesareana foram mais elevadas para mulheres de melhor nível socioeconômico, para as com pré-natal adequado, as primíparas, as multíparas e nas gestações duplas, sugerindo que essa indicação não se baseou somente em normas técnicas, mas também em razões não-médicas.

Abstract

PURPOSE: to determine the cesarean section (CS) rate in Campinas (SP) and to identify its risk factors.

METHODS: a cross-sectional study that analyzed data obtained from Live Birth Certificates in 2001. The dependent variable was the type of delivery and the independent variables were: mothers' characteristics and those related to their pregnancies, deliveries and to newborns. The assessment of the association among variables was performed through the $\chi^{2}$ test, and crude and adjusted odds ratio (OR) values were calculated. RESULTS: the CS rate was $54.9 \%$. The chances of having CS increased 1.9 times for women from 20-34 years old (adjOR-1.9; 95\% Cl: 1.7-2.1); 3.7 times for those over 35 years old (adjOR-3.8; 95\% Cl:3.2-4.5); 1.5 times for those who studied from 8-11 years (adjOR-1.5; 95\% Cl: 1.4-1.6); 2.5 times for those who studied more than 11 years (adjOR-2.6; 95\% Cl:2.2-2.9); 1.3 times for those who were married (adjOR-1.3; 95 \% Cl: 1.2-1.4); 1.6 times for those who had jobs (adjOR-1.6; 95\% Cl: 1.5-1.8); 1.2 times for who had good living conditions (adjOR-1 .2; 95\% Cl: 1.0-1.3); 2.2 times for primiparous (adjOR-2.2; 95\% Cl: 1.9-2.5), 1.6 times for multiparous (adjOR-1.6; 95\% Cl: 1.4-1.9) and 2.7 times in twin gestations (adjOR-2.7; 95\% Cl: 1.9-3.9). The women who had inadequate prenatal care were protected for CS (adjOR-0.6; 95\% Cl:0.5-0.7).CONCLUSION: the chance of having CS was greater among women with better socio-economic conditions, with adequate prenatal care, for primiparous, for multiparous and in twin gestations, suggesting that the basis for indication of cesarean sections were not restricted to clinical factors but influenced by non-medical reasons.

Correspondência:

Emília de Faria Carnie

Rua Serra Formosa, 66 - Jardim Paranapanem CEP 13100-268 - Campinas /SP - Brasil Fone: (19) 3255-6091 - E-mail: emiliac@fcm.unicamp.br

Recebido

22/05/2006

Aceito com modificacões

$28 / 11 / 2006$
Trabalho realizado no Departamento de Pediatria da Faculdade de Ciências Médicas da Universidade Estadual de Campinas - UNICAMP - Campinas (SP), Brasil.

Enfermeira Sanitarista do Departamento de Pediatria da Faculdade de Ciências Médicas da Universidade Estadual de Campinas - UNICAMP - Campinas (SP), Brasil.

2 Professora Assistente do Departamento de Pediatria da Faculdade de Ciências Médicas da Universidade Estadual de Campinas - UNICAMP - Campinas (SP), Brasil.

3 Professor Assistente do Departamento de Pediatria da Faculdade de Ciências Médicas da Universidade Estadual de Campinas - UNICAMP - Campinas (SP), Brasil. 


\section{Introdução}

O parto cesáreo é uma alternativa médica usada em situações em que as condições materno-fetais não favorecem o parto vaginal. Apesar do reconhecimento da contribuição dessa intervenção para uma melhor assistência à saúde e da segurança da cesárea moderna, é importante que sua indicação seja criteriosa, pois não é um procedimento inócuo e pode trazer riscos adicionais para a mãe e a criança ${ }^{1-3}$.

Estudos mostram maior risco de mortalidade e morbidade materna, como hemorragias, infecções puerperais, embolia pulmonar, riscos anestésicos, entre outros $^{1,2}$. Para o recém-nascido $(\mathrm{RN})$, há maior probabilidade de ocorrerem distúrbios respiratórios, icterícia fisiológica, prematuridade iatrogênica, hipoglicemia, anóxia, entre outros ${ }^{3,4}$. Além disso, há interferência no vínculo mãe-filho, o qual pode influenciar negativamente o aleitamento materno ${ }^{4}$. Adicionalmente, há maior consumo de recursos hospitalares, incorrendo em maiores custos decorrentes do procedimento, do maior tempo de internação e da morbidade conseqüente ${ }^{4,5}$.

Apesar de a Organização Mundial da Saúde (OMS) recomendar taxas de no máximo $15 \%$, vem se observando, desde o início da década de 70, uma tendência mundial para aumento deste tipo de parto. As taxas são bastante variáveis nos diversos países, sendo $13,5 \%$ na Holanda, $16 \%$ na Noruega e 16,3\% na Finlândia em 2002. No mesmo ano, a França teve 18,6\% de partos cesáreos, a Espanha, 23,4\% e a Itália, 36,1\% . O Reino Unido e os Estados Unidos, em 2002, apresentaram proporções de 21,6 e 26,1\%, respectivamente ${ }^{7}$. Na América Latina, um grande estudo ecológico mostrou que essas taxas foram muito elevadas em diversos países, pois somente sete dos 19 apresentaram taxas menores de $15 \%$ e nos restantes, onde ocorreram $81 \%$ dos partos, houve uma variação entre $16,8 \%$, na Colômbia, e 40,0\%, no México5.

O Brasil é hoje um dos países com maior ocorrência de cesarianas do mundo. Houve um aumento significativo com o passar dos anos, oscilando de $14,6 \%$ no início da década de 70 para 31,0\% na década de 804 . Em 1995, esta proporção foi para $35,5 \%$, e se mantém neste patamar com pequenas variações. Em 2001, a taxa média de cesarianas foi de $38,1 \%$, variando entre $17,4 \%$, no Amapá, e 49,4\%, no Rio de Janeiro. O Estado de São Paulo teve, em 2001, uma das maiores taxas do país $(49,1 \%)$ e a região de Campinas, uma das maiores do Estado (51,8\%). Na cidade de Campinas observa-se esta mesma tendência, com índices excessivamente superiores em relação ao recomendado pela OMS, apresentando aumento temporal nas últimas décadas ${ }^{8}$.

Há diversos critérios técnicos que indicam o parto cesáreo; no entanto, somente esses não são suficientes para justificar as altas taxas. No Brasil, como em outros países, diversos estudos têm mostrado associação entre o parto cesáreo e outros fatores não-clínicos, como os socioeconômicos (alta escolaridade materna, maior poder econômico ${ }^{9,10}$ e seguro de saúde privado ${ }^{10,11}$ ), os relacionados às características demográficas e reprodutivas (idade materna, primiparidade ${ }^{11}$, cesárea prévia ${ }^{12}$ ) e os relacionados aos serviços de saúde (maior freqüência ao pré-natal ${ }^{11}$, nos partos realizados em serviços privados ${ }^{10,11,13,14}$, por conveniência médica ${ }^{11,12,15}$, por falta de capacitação médica ${ }^{12}$, quando há admissão precoce da gestante ${ }^{12,15}$, por cesárea a pedido ${ }^{12,13}$, por fatores institucionais ${ }^{15}$ e por mudanças nas práticas obstétricas $\left.{ }^{16}\right)$. E, apesar dos altos índices, a literatura sugere que o decréscimo na mortalidade perinatal não foi influenciado pela taxa de cesariana, mas provavelmente pela melhora da assistência neonatal ${ }^{17}$. Além disso, nos estudos de opinião das mulheres sobre a preferência pelo tipo de parto, o argumento de que a grande maioria delas prefere o parto cesáreo ao vaginal não se confirmou ${ }^{14,18}$.

O presente estudo tem como objetivo conhecer a freqüência do parto cesáreo em Campinas (SP) em 2001, sua relação com as características maternas e dos $\mathrm{RN}$ bem como identificar fatores de risco para sua ocorrência.

\section{Métodos}

Realizou-se um estudo transversal, a partir de dados obtidos nas Declarações de Nascidos Vivos (DNVs) do Sistema de Informações sobre Nascidos Vivos (Sinasc), correspondentes aos partos de mulheres residentes em Campinas (SP), ocorridos no próprio município em 2001.

A cidade de Campinas, localizada a cerca de 100 $\mathrm{km}$ a noroeste da capital paulista, é sede de uma região metropolitana. É a terceira maior cidade do Estado, com uma população de 983.945 habitantes em 2001 (98,4\% residentes em área urbana) e tendo o oitavo Índice de Desenvolvimento Humano do Estado ${ }^{19}$. Com relação aos diferenciais nos níveis de qualidade de vida e de saúde da população, o indicador da Secretaria Municipal de Saúde, chamado Índice de Condição de Vida (ICV), apontou que a maioria das áreas de abrangência dos Centros de Saúde dos Distritos de Saúde (DS) Noroeste e Sudoeste pertencia ao grupo de pior ICV, e as do DS Leste, ao de melhor ICV. As áreas dos Centros de Saúde nos DS Norte e Sul estiveram mais homogeneamente distribuídas entre o melhor, médio e pior $\mathrm{ICV}^{19}$.

O perfil das mães e dos RNs foi identificado pelas características sociodemográficas maternas, relacionadas à gestação, ao parto e ao RN.

Para análise estatística, foi considerada variável dependente o tipo de parto (vaginal ou cesáreo). As 
variáveis independentes foram: DS de moradia (Norte, Sul, Leste, Noroeste e Sudoeste), idade materna ( $<20$ anos, 20 a 34 anos e $\geq 35$ anos), escolaridade (até sete, de oito a 11 e 12 ou mais anos de estudo concluídos), ocupação (com ou sem ocupação), situação conjugal (com ou sem companheiro), tipo de gestação (única, dupla e $\geq$ três), número de consultas de pré-natal ( $<$ sete e $\geq$ sete), número de filhos vivos tidos em gestações anteriores (zero, um, dois e $\geq$ três), número de filhos mortos (zero, um e $\geq$ dois) e peso do $\mathrm{RN}$ ao nascimento em gramas (<2.500, 2.500 a 2.999 e $\geq 3.000)$.

Estudou-se a associação entre a variável dependente e as independentes, utilizando-se o teste do $\chi^{2}$ e valores de odds ratio (OR) brutos. Empregou-se o modelo de regressão logística (método stepwise backward) usando o software SPPS 11.0, com o objetivo de obterem-se os valores de OR ajustados (ORaj).

As categorias escolhidas como referência foram as de menor risco esperado para parto cesáreo (DS Noroeste - o de pior ICV, mãe com menos de 20 anos idade, com até sete anos de estudo concluídos, sem ocupação, sem companheiro e com três filhos ou mais). Tais grupos são comumente de usuárias de serviços financiados pelo Sistema Único de Saúde (SUS), que impõe limitações para a ocorrência de partos cesáreos em 30\%. As maiores taxas são provenientes, principalmente, de partos ocorridos em serviços privados ${ }^{20}$. A variável número de consultas de pré-natal teve como referência a categoria de maior ou igual a sete consultas, considerando-se que, durante o atendimento pré-natal, a gestante poderá discutir e receber orientações sobre a forma mais adequada e segura para a resolução de sua gestação.

Esse trabalho foi aprovado pelo Comitê de Ética da Faculdade de Ciências Médicas da Universidade Estadual de Campinas (FCM/UNICAMP).

\section{Resultados}

Dos 14.444 nascimentos ocorridos em Campinas em 2001, obteve-se informação sobre o tipo de parto de 14.439, sendo que a taxa de cesarianas foi de 54,9\%. As taxas variaram de acordo com os diferentes DS do município, tendo o Noroeste e o Sudoeste as menores proporções de cesárea, e os DS Norte, Sul e Leste, os percentuais acima da média do município, sobretudo no DS Leste, onde este índice foi de 63,5\%.

As chances de cesariana foram maiores para as mulheres maiores de 20 anos, as que tinham ocupação, as com companheiro, para as moradoras do DS Norte, do Sul e do Leste, para as que estudaram entre 8 a 11 anos, e para as com 12 anos e mais de estudos (Tabela 1).

Tabela 1 - Distribuição de freqüência e valores dos odds ratio brutos do tipo de parto em relação à situação conjugal, ao Distrito de Saúde, à escolaridade, à ocupação e à idade materna.

\begin{tabular}{|c|c|c|c|c|c|c|}
\hline \multirow{2}{*}{ Categorias de análise } & \multirow{2}{*}{$\mathbf{N}$} & \multicolumn{2}{|c|}{ Cesćrea } & \multirow{2}{*}{ OR } & \multirow{2}{*}{ IC $95 \%$} & \multirow[b]{2}{*}{$\mathbf{p}$} \\
\hline & & n & $\%$ & & & \\
\hline \multicolumn{7}{|l|}{ Situação conjugal } \\
\hline Sem companheiro & 5.166 & 2.335 & 45,2 & 1,00 & & \\
\hline Com companheiro & 9.238 & 5.570 & 60,3 & 1,83 & $1,71 \cdot 1,96$ & $<0,01$ \\
\hline \multicolumn{7}{|l|}{ Distrito de Saúde } \\
\hline Norte & 2.365 & 1.366 & 57,8 & 1,44 & $1,28-1,61$ & $<0,01$ \\
\hline Sul & 3.682 & 2.029 & 55,1 & 1,29 & $1,16 \cdot 1,43$ & \\
\hline Leste & 2.507 & 1.592 & 63,5 & 1,83 & $1,63 \cdot 2,05$ & \\
\hline Noroeste & 2.553 & 1.245 & 48,8 & 1,00 & & \\
\hline Sudoeste & 3.332 & 1.692 & 50,8 & 1,08 & $0,98-1,20$ & \\
\hline \multicolumn{7}{|l|}{ Escolaridade da mãe } \\
\hline Até 7 & 5.386 & 2.193 & 40,7 & 1,00 & & \\
\hline 8 a 11 & 6.723 & 3.946 & 58,7 & 2,07 & $1,92 \cdot 2,23$ & $<0,01$ \\
\hline 12 e mais & 2.157 & 1.734 & 80,4 & 5,97 & $5,29-6,74$ & \\
\hline \multicolumn{7}{|l|}{ Ocupação } \\
\hline Sem ocupação & 8.734 & 3.939 & 45,1 & 1,00 & & \\
\hline Com ocupação & 5.687 & 3.972 & 69,8 & 2,82 & $2,63-3,03$ & $<0,01$ \\
\hline \multicolumn{7}{|l|}{ Idade materna } \\
\hline$<20$ & 2.562 & 928 & 36,2 & 1,00 & & \\
\hline $20 \vdash 35$ & 10.364 & 5.931 & 57,2 & 2,36 & $2,15-2,58$ & $<0,01$ \\
\hline$\geq 35$ & 1.511 & 1.064 & 70,4 & 4,19 & $3,65-4,82$ & \\
\hline
\end{tabular}

N: freqüência absoluta; \%: freqüência relativa; OR: odds ratio; IC 95\%: intervalo de confiança de 95\%; p: probabilidade do teste do $\chi^{2}$.

Fonte: Secretaria Municipal de Saúde - CoVISA (Coordenadoria de Vigilância e Saúde Ambiental)/Departamento de Informação e Informática Banco de Dados: Sistema de Informação sobre Nascidos Vivos de Campinas, janeiro a dezembro, 2001. 
Foram também encontradas associações entre parto cesáreo e RNs provenientes de gestação dupla. As mulheres que tinham um ou mais filhos mortos, as primíparas, ou as multíparas com um ou dois filhos em gestações anteriores estiveram mais expostas à cesárea. As que fizeram menos que sete consultas de pré-natal foram protegidas do parto cesáreo (Tabela 2).

Para a análise de regressão logística, foram selecionadas as variáveis idade, situação conjugal, DS, ocupação, escolaridade materna, número de filhos vivos e mortos, tipo da gestação e número de consultas de pré-natal (Tabelas 3 e 4).

Observou-se que as mulheres de 20 a 34 anos $(\mathrm{ORaj}=1,92 ; \mathrm{IC}$ a $95 \%: 1,72-2,14)$, as com idade igual ou superior a 35 anos (ORaj=3,75; IC a 95\%:3,15$4,47)$, as que estudaram de 8 a 11 anos (ORaj=1,50; IC a 95\%:1,38-1,62) ou 12 anos ou mais (ORaj=2,55; IC a 95\%: 2,20-2,95), as que tinham companheiro (ORaj=1,33; IC a 95\%:1,23-1,44), as que trabalhavam fora do lar (ORaj=1,64; IC a 95\%:1,51-1,79) e as que residiam no DS Norte (ORaj=1,16; IC a 95\%:1,021,32), no Sul (ORaj=1,19; IC a 95\%:1,06-1,34) e no Leste (ORaj=1,16; IC a 95\%:1,01-1,32) tiveram maior chance de serem submetidas ao parto cesáreo.

Da mesma forma, as primíparas (ORaj $=2,17$; IC a 95\%:1,87-2,50) ou as multíparas com um (ORaj: 1,70;
IC 95\%: 1,48-1,96) ou dois filhos (ORaj=1,58; IC a 95\%:1,35-1,85) em gestações anteriores apresentaram maior risco para parto cesáreo.

As crianças provenientes de gestações duplas (ORaj=3,14; IC a 95\%:2,22-4,42) também tiveram maior chance de nascerem por cesariana.

As mulheres que fizeram menos consultas de prénatal apresentaram o menor risco para parto cesáreo (ORaj=0,59; IC a 95\%:0,54-0,65).

\section{Discussão}

O Sinasc em Campinas foi implantado no início da década de 90, conforme orientação do Ministério da Saúde, e, atualmente, sob coordenação da Secretaria Municipal de Saúde, apresenta uma proporção de captação de $99,1 \%$, considerado como nível de cobertura excelente $^{21}$. O percentual de preenchimento dos itens das DNVs foi superior a $99 \%$. O número de consultas de pré-natal foi o dado menos registrado, com 9,4\% de declarações sem esta informação. Com relação à fidedignidade dos dados presentes nas declarações, já foi demonstrado que, para a maioria das variáveis, os registros são confiáveis ${ }^{21}$.

O percentual de cesarianas encontrado para o município em 2001 foi mais do que o triplo do recomendado

Tabela 2 - Distribuição de frequiência e valores do odds ratio brutos do tipo de parto em relação ao número de filhos nascidos vivos e mortos, ao número de consultas de pré-natal e ao peso de nascimento.

\begin{tabular}{|c|c|c|c|c|c|c|}
\hline \multirow{2}{*}{ Categorias de análise } & \multirow{2}{*}{$\mathbf{N}$} & \multicolumn{2}{|c|}{ Cesárea } & \multirow{2}{*}{ OR } & \multirow{2}{*}{ IC $95 \%$} & \multirow{2}{*}{$\mathbf{p}$} \\
\hline & & n & $\%$ & & & \\
\hline \multicolumn{7}{|l|}{ Filhos mortos } \\
\hline 0 & 13.241 & 7.193 & 54,3 & 1,00 & & \\
\hline 1 & 970 & 585 & 60,3 & 1,28 & $1,11-1,46$ & $<0,01$ \\
\hline$\geq 2$ & 214 & 137 & 64,0 & 1,49 & $1,12 \cdot 2,00$ & \\
\hline \multicolumn{7}{|l|}{ Filhos vivos } \\
\hline 0 & 6.582 & 3.894 & 59,2 & 2,41 & $2,14 \cdot 2,71$ & $<0,01$ \\
\hline 1 & 4.416 & 2.494 & 56,5 & 2,16 & $1,91-2,44$ & \\
\hline 2 & 1.939 & 971 & 50,1 & 1,67 & $1,45 \cdot 1,92$ & \\
\hline$\geq 3$ & 1.494 & 561 & 37,6 & 1,00 & & \\
\hline \multicolumn{7}{|l|}{ Consultas de pré-natal } \\
\hline$<7$ & 3.353 & 1.324 & 39,5 & 0,42 & $0,39 \cdot 0,45$ & $<0,01$ \\
\hline$\geq 7$ & 9.732 & 5.924 & 60,9 & 1,00 & & \\
\hline \multicolumn{7}{|l|}{ Tipo de gestação } \\
\hline Única & 14.168 & 7.713 & 54,4 & 1,00 & & \\
\hline Dupla & 255 & 201 & 78,8 & 3,12 & $2,28-4,26$ & $<0,01$ \\
\hline$\geq 3$ & 16 & 11 & 68,8 & 1,84 & $0,59-6,07$ & \\
\hline \multicolumn{7}{|l|}{ Peso de nascimento (g) } \\
\hline$<2.500$ & 1.316 & 751 & 57,1 & 1,03 & $0,92 \cdot 1,16$ & \\
\hline $2.500+3.000$ & 3.719 & 1.883 & 50,6 & 0,80 & $0,74-0,86$ & $<0,01$ \\
\hline$\geq 3.000$ & 9.404 & 5.291 & 56,3 & 1,00 & & \\
\hline
\end{tabular}

N: freqüência absoluta; \%: freqüência relativa; OR: odds ratio; IC 95\%: intervalo de confiança de 95\%; p: probabilidade do teste do $\chi^{2}$.

Fonte: Secretaria Municipal de Saúde - CoVISA (Coordenadoria de Vigilância e Saúde Ambiental)/Departamento de Informação e Informática

Banco de Dados: Sistema de Informação sobre Nascidos Vivos de Campinas, janeiro a dezembro, 2001. 
pela OMS, acompanhando a tendência de aumento com os anos. No início da década de 70, este índice era de $27,1 \%$ e chegou, na década de 1990 , a 53,4\%. Desde então, vem se mantendo acima de 50,0\%

Os achados do presente estudo indicam que a chance para parto cesáreo foi maior para as mulheres com companheiro, as moradoras das regiões com melhores condições de vida, as com melhor nível educacional, as que referiram ter ocupação fora do lar, as maiores de 20 anos e as que freqüentaram adequadamente o serviço de pré-natal.

Esses resultados concordam com os observados em estudo realizado em uma maternidade de Campinas (SP), no período de 1971 a 1995. O maior risco de cesáreas apresentou-se para as mulheres de 20 a 34 anos, com companheiro, que trabalhavam fora, que não eram previdenciárias e que freqüentaram os serviços de pré-natal. Esses autores também apontaram para uma tendência de diminuição do peso de nascimento dos RNs que nasceram por cesárea e supuseram que essa

Tabela 3 - Valores de odds ratio ajustados do tipo de parto associados a variáveis sociodemográficas materna em Campinas (SP), 2001.

\begin{tabular}{|c|c|c|c|}
\hline Variável & OR bruta & OR ajustada & IC $95 \%$ \\
\hline \multicolumn{4}{|l|}{ Situação conjugal } \\
\hline Com companheiro & 1,83 & 1,33 & $1,23 \cdot 1,44$ \\
\hline \multicolumn{4}{|l|}{ Distrito de Saúde } \\
\hline Norte & 1,44 & 1,16 & $1,02 \cdot 1,32$ \\
\hline Sul & 1,29 & 1,19 & $1,06-1,34$ \\
\hline Leste & 1,83 & 1,16 & $1,01-1,32$ \\
\hline \multicolumn{4}{|l|}{ Escolaridade da mãe } \\
\hline 8 a 11 & 2,07 & 1,50 & $1,38 \cdot 1,62$ \\
\hline 12 e mais & 5,97 & 2,55 & $2,20-2,95$ \\
\hline \multicolumn{4}{|l|}{ Idade materna } \\
\hline $20-35$ & 2,36 & 1,92 & $1,72 \cdot 2,14$ \\
\hline$\geq 35$ & 4,19 & 3,75 & $3,15-4,47$ \\
\hline \multicolumn{4}{|l|}{ Ocupação } \\
\hline Com ocupação & 2,82 & 1,64 & $1,51 \cdot 1,79$ \\
\hline
\end{tabular}

OR: odds ratio; IC 95\%: intervalo de confiança de $95 \%$.

Tabela 4 - Valores de odds ratio ajustados do tipo de parto associados a variáveis gestacionais em Campinas (SP) 2001.

\begin{tabular}{|c|c|c|c|}
\hline Variável & OR bruta & OR ajustada & IC $95 \%$ \\
\hline \multicolumn{4}{|c|}{ Número de fillhos } \\
\hline 0 & 2,41 & 2,17 & $1,87-2,50$ \\
\hline 1 & 2,16 & 1,70 & $1,48 \cdot 1,96$ \\
\hline 2 & 1,67 & 1,58 & $1,35 \cdot 1,85$ \\
\hline \multicolumn{4}{|c|}{ Consultas de pré-natal } \\
\hline$<7$ & 0,42 & 0,59 & $0,54-0,65$ \\
\hline \multicolumn{4}{|c|}{ Tipo de gestação } \\
\hline Dupla & 3,12 & 3,14 & $2,22-4,42$ \\
\hline
\end{tabular}

OR: odds ratio; IC 95\%: intervalo de confiança de $95 \%$. conduta poderia estar interrompendo o potencial de crescimento intra-uterino $^{22}$. Resultado semelhante foi encontrado em estudo sobre o tipo de parto de acordo com a categoria de internação, que verificou que a incidência de cesariana foi crescente à medida que se elevou o padrão social das gestantes, sem que houvesse correspondência com o risco obstétrico ${ }^{13}$.

Outros estudos também verificaram a maior ocorrência de cesariana em grupos de menor risco obstétrico, como as mulheres de estratos sociais mais elevados, com maior escolaridade, maior renda, maior idade, que fizeram mais consultas de pré-natal e que foram atendidas em serviços privados e conveniados $5,10,11,13$. Esses achados sugerem que a decisão de realizar o parto cirúrgico não se baseou somente em critérios técnicos e mostram que esse tipo de parto tem adquirido um caráter de bem de consumo que pode ser utilizado por quem possa custeá-lo ${ }^{23}$.

Encontrou-se também associação entre parto cesáreo e primíparas, multíparas e gestações duplas, do mesmo modo que observado em outros estudos.

Em estudo realizado em um hospital universitário de Campinas (SP) observou-se a maior ocorrência de partos cesáreos em nulíparas com baixo risco obstétri$\mathrm{co}^{3}$. No Rio de Janeiro, também foi encontrada essa associação, destacando ser esse fato preocupante, pois a cesárea prévia está entre as principais indicações médicas para partos cirúrgicos subseqüentes, embora haja possibilidade de realização de parto vaginal nessa situação, se houver condições materno-fetais favoráveis ${ }^{12,24}$. Em uma cidade do interior de São Paulo, verificou-se que a quase totalidade das mulheres com dois ou mais filhos que fizeram cesariana no primeiro parto também o fizeram no segundo ${ }^{10}$, e, em estudo realizado nos Estados Unidos, foi encontrado que mais da metade do recente aumento nas taxas de cesariana foi devido ao aumento das cesarianas em primíparas, o que poderá, no futuro, levar a um grande grupo de mulheres para quem cesáreas repetidas serão a norma ${ }^{16}$.

$\mathrm{Na}$ população estudada, houve dificuldade de analisar os motivos para a ocorrência de cesáreas em multíparas, uma vez que os dados do Sinasc não apresentam informações a respeito do tipo dos partos anteriores. Foi encontrada em Ribeirão Preto (SP) maior chance desse tipo de parto para qualquer número de filhos, supondo que a laqueadura possa ser uma das principais razões dessa ocorrência ${ }^{11}$.

Quanto aos gemelares, há indicações de cesarianas em determinadas situações de apresentações fetais e intercorrências obstétricas ${ }^{24}$. Por não ser motivo dessa investigação, esse tipo de indicação não foi avaliada, sendo necessárias pesquisas que abordem esse tema especificamente. Em 
estudo realizado em Recife (PE) verificou-se que a chance de cesariana para partos de gemelares era 8,3 vezes maior do que para partos únicos, possivelmente devido à prática menos freqüente de versão fetal, realizadas no serviço. Nesse mesmo trabalho a primiparidade também constituiu fator de risco para parto operatório ${ }^{25}$.

Este estudo mostrou que, assim como em outras localidades do país, a ocorrência de cesariana em Campinas, em 2001, está muito além do recomendado por órgãos nacionais e internacionais. A análise dos fatores de risco sugerem que sua indicação pareceu não se basear somente em normas técnicas, mas em diversas razões como as apontadas em outros estudos, tais como insegurança médica no manejo do parto vaginal ${ }^{26} \mathrm{e}$ medo da responsabilização jurídica ${ }^{27}$; crença de que este tipo de parto é um procedimento seguro, sem sofrimento e com maior conforto para a mãe ; noção de que esta intervenção representa assistência de melhor qualidade ${ }^{9}$, por maior conveniência médica ${ }^{11}$; pela possibilidade de laqueadura ${ }^{11,18}$; por desinformação e falta de participação das mulheres nas decisões relacionadas à gravidez; por falta de comunicação entre médico ${ }^{26}$ e paciente, dentre outros.

Cabe ressaltar que, embora haja a idéia de que as mulheres tenham preferência pelo parto cesáreo por receio da dor do parto vaginal, por razões estéticas ou por temor de prejuízo na vida sexual, vários autores mostraram que o parto vaginal é esperado pela maioria das gestantes, tanto as usuárias do SUS quanto as dos serviços privados ${ }^{14}$. A opção pelo parto vaginal é justificada por elas pela recuperação pós-parto mais rápida ${ }^{14,18,28}$, pelo medo do parto cesáreo e pela dor provocada pela incisão cirúrgica ${ }^{15,18,26,28}$. Aquelas que referiram desejar a cesariana foi pela possibilidade de esterilização $^{14,28}$, em situações concretas de dor, ou por medo da reação dos profissionais às suas queixas durante o trabalho de parto ${ }^{28}$. Foi observado também que muitas dessas mulheres desconheciam o motivo da indicação da cesariana ${ }^{29}$, sugerindo que há indução médica para a realização do parto cirúrgico ${ }^{10}$.

Uma limitação desse estudo foi a impossibilidade de avaliar todas as situações que poderiam determinar o parto cesáreo, visto que a análise baseou-se somente nas variáveis das DNVs do Sinasc. No entanto, os resultados encontrados poderão nortear a implementação de políticas de saúde materno-infantil, de modo que a assistência à gestante seja realizada dentro de critérios técnicos, éticos e humanitários, proporcionando maior segurança e menor índice de complicações.

\section{Referências}

1. Ramos JGL, Martins-Costa S, Vettorazzi-Stuczynsky J, Brietzke E. Morte materna em hospital terciário do Rio Grande do Sul: um estudo de 20 anos. Rev Bras Ginecol Obstet. 2003; 25(6):431-6.

2. Villar J, Valladares E, Wojdyla D, Zavaleta N, Carroli G, Velazco $A$, et al. Caesarean delivery rates and pregnancy outcomes: the 2005 WHO global survey on maternal and perinatal health in Latin America. Lancet. 2006; 367(9525): 1819-29.

3. Telini DMTZ. Cesáreas e partos normais em gestantes com baixo risco obstétrico: características maternas e repercussões neonatais [tese]. Campinas: Universidade Estadual de Campinas. Faculdade de Ciências Médicas; 2000.

4. Faúndes A, Cecatti JG. A operação cesárea no Brasil: incidência, tendências, causas, conseqüências e propostas de ação. Cad Saúde Pública. 1991; 7(2):150-73.

5. Belizan JM, Althabe F, Barros FC, Alexander S. Rates and implications of caesarean sections in Latin America: ecological study. BM. 1999; 319(7222):1397-400.

6. World Health Organization [homepage on the Internet]. European health for all database 2002 [cited 2006 Out 12]. Available from: http://data.euro.who.int/hfadb

7. Martin JA, Hamilton BE, Sutton PD, Ventura SJ, Menacker, F, Munson ML. Births: final data for 2002. Natl Vital Stat Rep [serial on the Internet]. 2003 [cited 2006 Out 12];52(10). Available from: http://www.cdc.gov/nchs/data/nvsr/nvsr52/nvsr52_10

8. Ministério da Saúde [sítio na Internet]. Sistema de Informações de Nascidos Vivos (Sinasc). 2003 [citado 2004 Maio 12 ]. Disponível em: http://www.funasa.gov.br/sis/sisO2htm.2003
9. Behague DP, Victora CG, Barros FC. Consumer demand for caesarean sections in Brazil: informed decision making, patient choice, or social inequality? A population based birth cohort study linking ethnographic and epidemiological methods. BM. 2002; 324(7343):942-5

10. Moraes MS, Goldenberg P. Cesáreas: um perfil epidêmico. Cad Saúde Pública. 2001; 17(3):509-19.

11. Gomes UA, Silva AA, Bettiol H, Barbieri MA. Risk factors for the increasing caesarean section rate in Southeast Brazil: a comparison of two birth cohorts, 1978-1979 and 1994. Int J Epidemiol. 1999; 28(4):687-94.

12. D'Orsi E, Chor D, Giffin K, Angulo-Tuesta A, Barbosa GP, Gama Ade $S$, Reis AC. Factors associated with cesarean sections in a public hospital in Rio de Janeiro, Brazil. Cad Saúde Pública. 2006; 22(10):2067-78

13. Yazlle MEHD, Rocha JSY, Mendes MC, Patta MC, Marcolin AC, Azevedo GD. Incidência de cesáreas segundo fonte de financiamento da assistência ao parto. Rev Saúde Pública. 2001; 35(2):202-6.

14. Potter JE, Berquo E, Perpetuo IH, Leal OF, Hopkins K, Souza $M R$, Formiga MC. Unwanted caesarean sections among public and private patients in Brazil: prospective study. BM. 2001; 323(7322): $1155-8$.

15. Bailit JF, Love TE, Mercer B. Rising cesarean rates: are patients sicker? Am J Obstet Gynecol. 2004; 191 (3):800-3.

16. Declercq E, Menacker F, Macdorman M. Maternal risk profiles and the primary cesarean rate in the United States, 1991-2002. Am J Public Health. 2006; 96(5):867-72. 
17. Duarte G, Coltro PS, Bedone RV, Nogueira AA, Gelonezzi GM, Franco $L$. Trends in the modes of delivery and their impact on perinatal mortality rates. Rev Saúde Pública. 2004; 38(3):379-84.

18. Barbosa GP, Giffin K, Angulo-Tuesta A, Gama AS, Chor D, D'Orsi $\mathrm{E}$, et al. Parto cesáreo: quem o deseja? Em quais circunstâncias? Cad Saúde Pública. 2003; 19(6):1611-20.

19. Prefeitura Municipal de Campinas [sítio na Internet]. Informação em Saúde: saúde em números. 2001 [citado 2004 Set 16]. Disponível em: http://www.campinas.sp.gov.br/saude

20. Duarte EC, Schneider MC, Paes-Souza R, Ramalho WM, Sardinha LMV, Silva Junior JB, et al. Epidemiologia das desigualdades no Brasil: um estudo exploratório. Brasília: Athalaia; 2002.

21. Mello Jorge MHP, Gotlieb SLD, Oliveira H. O Sistema de Informação sobre Nascidos Vivos: primeira avaliação dos dados brasileiros. Inf Epidemiol SUS. 1996; 2:15-48.

22. Bicalho GG, Barros Filho AA. Parto cesáreo e o peso ao nascer na maternidade de Campinas, SP: 1971 - 1995. Pediatria (São Paulo). 2001; 23(3):223-31.

23. Freitas PF, Drachler ML, Leite JCC, Grassi PR. Desigualdade social nas taxas de cesariana em primíparas no Rio Grande do Sul. Rev Saúde Pública. 2005; 39(5):761-7.
24. Camano L, Souza E. Assistência ao parto e à tocurgia. Manual de orientação FEBRASGO. São Paulo: FEBRASGO; 2002.

25. Cabral SALCS, Costa CFF, Cabral Júnior SF. Correlação entre a idade materna, paridade, gemelaridade, síndrome hipertensiva e ruptura prematura de membranas e a indicação de parto cesáreo. Rev Bras Ginecol Obstet. 2003; 25(10):739-44.

26. Faúndes A, Pádua KS, Osis MD, Cecatti JG, Sousa MH. Opinião de mulheres e médicos brasileiros sobre a preferência pela via de parto. Rev Saúde Pública. 2004; 38(4):488-94.

27. Dias MAB, Deslandes SF. Cesarianas: percepção de risco e sua indicação pelo obstetra em uma maternidade pública no município do Rio de Janeiro. Cad Saúde Pública. 2004; 20(1):109-16

28. Hotimsky SN, Rattner D, Venâncio SI, Bógus CM, Miranda MM. O parto como eu vejo... ou como eu o desejo? Expectativas de gestantes, usuárias do SUS, acerca do parto e da assistência obstétrica. Cad Saúde Pública. 2002; 18(5):1303-11.

29. Oliveira SMV, Gonzalez Riesco ML, Rosas Miya CF, Vidotto P. Tipo de parto: expectativas das mulheres. Rev Latinoam Enfermagem. 2002; 10(5):667-74. 